Steep capillary-gravity waves in oscillatory shear-driven flows

Jalikop, Shreyas V. and Juel, Anne

2009

MIMS EPrint: 2010.79

Manchester Institute for Mathematical Sciences

School of Mathematics

The University of Manchester

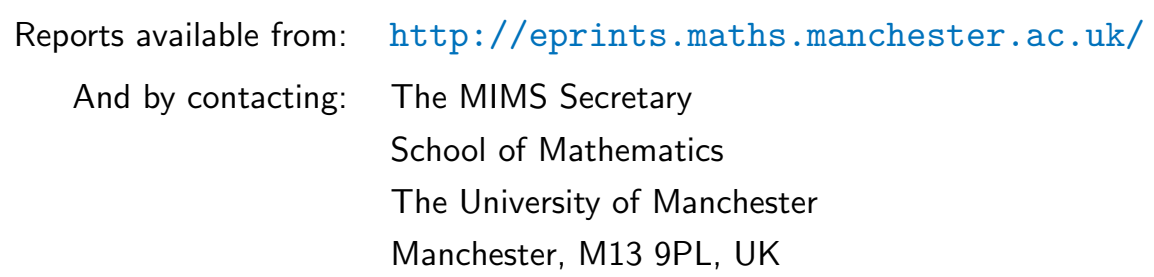

ISSN 1749-9097 


\title{
Steep capillary-gravity waves in oscillatory shear-driven flows
}

\author{
SHREYAS V. JALIKOP† AND ANNE JUEL \\ School of Mathematics and Manchester Centre for Nonlinear Dynamics, The University \\ of Manchester, Manchester M13 9PL, UK
}

(Received 18 July 2008; revised 7 August 2009; accepted 10 August 2009; first published online 10 November 2009)

We study steep capillary-gravity waves that form at the interface between two stably stratified layers of immiscible liquids in a horizontally oscillating vessel. The oscillatory nature of the external forcing prevents the waves from overturning, and thus enables the development of steep waves at large forcing. They arise through a supercritical pitchfork bifurcation, characterized by the square root dependence of the height of the wave on the excess vibrational Froude number ( $W$, square root of the ratio of vibrational to gravitational forces). At a critical value $W_{c}$, a transition to a linear variation in $W$ is observed. It is accompanied by sharp qualitative changes in the harmonic content of the wave shape, so that trochoidal waves characterize the weakly nonlinear regime, but 'finger'-like waves form for $W \geqslant W_{c}$. In this strongly nonlinear regime, the wavelength is a function of the product of amplitude and frequency of forcing, whereas for $W<W_{c}$, the wavelength exhibits an explicit dependence on the frequency of forcing that is due to the effect of viscosity. Most significantly, the radius of curvature of the wave crests decreases monotonically with $W$ to reach the capillary length for $W=W_{c}$, i.e. the lengthscale for which surface tension forces balance gravitational forces. For $W<W_{c}$, gravitational restoring forces dominate, but for $W \geqslant W_{c}$, the wave development is increasingly defined by localized surface tension effects.

\section{Introduction}

Nonlinear waves in fluids are associated with a rich variety of dynamics that often underpin important natural phenomena. Examples range from internal solitary-like waves that are ubiquitous features of coastal oceans (Helfrich \& Melville 2006) to the surface ocean spectra, whose interpretation relies on the nonlinear interaction between surface water waves and wind (Phillips 1988). Stokes' early analysis (Stokes 1847) of the shape of gravity waves showed that above infinitesimal amplitudes, the free-surface wave that results from the balance between inertial and restoring gravity forces, adopts the shape of a trochoid, with steeper crests and flatter troughs. This solution, which omits surface tension, converges for all amplitudes less than the critical value at which the wave-crest curvature becomes infinite (Kraskovskii 1960). As suggested by Stokes, the included angle of the crest at this critical point is $120^{\circ}$, and its longitudinal velocity becomes equal to the phase speed, so that the wave breaks. When the restoring force is due to surface tension rather than gravity, an exact nonlinear solution first derived by Crapper (1957), describes progressive capillary waves of arbitrary amplitudes. Unlike gravity waves, the trough of a capillary wave

$\dagger$ Email address for correspondence: sjalikop@ma.man.ac.uk 
is sharper than its crest (inverted trochoid). Surface tension prevents the trough curvature from becoming infinite, and for a critical wave height, the free surface just touches itself and entrains an air bubble at the end of each trough.

The dispersion relation for small-amplitude capillary-gravity waves (linear waves) indicates that surface tension and gravity are the dominant restoring forces at large and small wavenumbers, respectively (Craik 1985). However, for finite wave amplitudes (nonlinear waves), surface tension effects can dominate irrespective of the wavenumber of the primary wave through mechanisms such as localized surface tension effects in steep waves and the resonance interaction mechanism (RIM). In steep waves where the curvature distribution is highly non-uniform, surface tension forces, which tend to limit the curvature to finite values, can act locally to produce features such as increased wave height (Taylor 1953) and ripple formation on progressive water waves (Cox 1958). Taylor's observation of wave heights larger than those predicted for standing gravity waves was shown to be due to surface tension effects by Schultz et al. (1998), as surface tension prevents a curvature singularity at the crest of the wave when its height reaches and then exceeds the gravity wave limit. By performing a stability analysis of progressive gravity waves near their maximum amplitude, Longuet-Higgins (1963) showed that the localization of surface tension effects near the wave crests produces a train of ripples on the forward face of the wave that is sustained by drawing energy from the gravity wave. However, surface tension can also exercise considerable influence at small to moderate wave steepness by altering the resonance conditions through nonlinear interactions between different wave modes, as exemplified by Wilton's ripples (McGoldrick 1970b). Moreover, localized surface tension effects and resonant interaction mechanisms can act together to produce features such as the Wilton 'dimple' observed by Jiang, Perlin \& Schultz (1998) on steep gravity-capillary waves.

In this paper, we investigate the growth to large amplitudes of progressive capillarygravity waves that form at the interface between two immiscible liquids through a Kelvin-Helmholtz (K-H) instability, and interpret the development of the waves in terms of surface tension effects. We are not aware of any previous studies of surface tension effects in steep shear-driven waves, as the wave crests generally bend with the basic stream (Drazin 1970), so that the waves become unstable at moderate amplitudes. The influence of shear on interfacial waves was addressed by Thorpe (1978) in two-layer experiments in miscible liquids, where he observed the transition from narrow (broad) troughs (crests) to markedly broader troughs than crests as the interfacial shear was reduced. The transition point, however, could not be determined accurately because of limited wave heights, beyond which a K-H instability occurred locally near the crests, leading to roll up and eventually to wave breaking and mixing. In the presence of surface tension between immiscible layers of counter-flowing liquids, Hou, Lowengrub \& Shelley's (1997) computations have shown that roll-up is suppressed, allowing the interface to form long 'fingers' of one liquid penetrating into the other. These 'fingers' are susceptible to overturning and form spirals for larger forcing. Hence, previous studies on shear-driven progressive interfacial waves have been limited to small amplitudes beyond which the waves break or roll-up either due to local shear instabilities or overturning of crests. Possibly due to this reason, the study of surface tension effects has been limited to large-amplitude standing waves (Taylor 1953; Schultz et al. 1998), rather than progressive waves. We prevent the overturning of crests by driving waves at the interface between immiscible liquids with oscillatory shear, and thus, we are able to generate large-amplitude progressive capillary-gravity waves. 
When a rectangular vessel containing two immiscible liquids of different densities in a stably stratified configuration is oscillated horizontally, the differential acceleration of the two liquids results in a counterflow that generates oscillatory interfacial shear. Capillary-gravity waves were first observed above a critical forcing acceleration by Wolf (1969), and their onset has subsequently been studied experimentally by Wunenburger et al. (1999), Ivanova, Kozlov \& Evesque (2001), González-Viñas \& Salán (1994) and Talib, Jalikop \& Juel (2007). They are often referred to as 'frozen waves' (FWs) because they appear motionless in the reference frame of the oscillating vessel for small enough wave amplitudes. In the reference frame of the laboratory, however, they can be considered as progressive waves with a time-periodic phase speed. In the inviscid limit of large frequencies, $\omega=2 \pi f$, and vanishing amplitudes of forcing $a$, Lyubimov \& Cherepanov (1987) predicted that the interface between two liquid layers, each of thickness $h$, becomes linearly unstable to a sinusoidal disturbance of dimensionless wavenumber $k$ when the vibrational Froude number, $\widehat{W}=a \omega / \sqrt{(g h)}$, where $g$ is the acceleration due to gravity, exceeds a critical value so that

$$
\widehat{W}^{2} \geqslant \frac{1}{2} W_{\gamma}\left(\frac{k}{k_{\gamma}}+\frac{k_{\gamma}}{k}\right) \tanh k
$$

with

$$
W_{\gamma}=\frac{\left(1+\rho_{2} / \rho_{1}\right)^{3}}{\rho_{2} / \rho_{1}\left(1-\rho_{2} / \rho_{1}\right)} k_{\gamma}^{-1} \quad \text { and } \quad k_{\gamma}=\frac{h}{l_{c}},
$$

where $\rho_{i}(i=1,2)$ is the density of the lower and upper layers, respectively. For layer heights $h>\sqrt{3} l_{c}$, where $l_{c}=\sqrt{\gamma /\left(\left(\rho_{1}-\rho_{2}\right) g\right)}$ is the capillary length and $\gamma$ the interfacial tension, the critical wavelength is determined by the capillary length, $\lambda_{c}=2 \pi / k_{\gamma}$. Thus, in the absence of viscosity, the capillary length is the natural length scale of the instability. The onset relation given by (1.1) is analogous to that of the classical K-H instability (Chandrasekhar 1981), where the short and longwavelength perturbations are suppressed by the restoring effects of surface tension and gravitational forces, respectively. The Froude number $(\widehat{W})$, which is a square root measure of vibrational to gravitational forces, is analogous to the dimensionless velocity difference across the interface in the classical K-H flow. Although both inviscid (Khenner et al. 1999) and viscous (Talib \& Juel 2007) models predict parametric modes of instability at finite amplitudes and frequencies of forcing, only the $\mathrm{K}-\mathrm{H}$ mode (zeroth order parametric mode) occurs for experimentally realizable parameters, where the ratio of kinematic viscosities $N=v_{2} / v_{1}$ (with the subscripts 1 and 2 referring to the lower and upper layers, respectively), is large and the interfacial tension is small (Talib et al. 2007; Yoshikawa (2006)). The presence of viscosity introduces two additional length scales, $\delta_{i}=\sqrt{2 v_{i} / \omega},(i=1,2)$, which are widely different when the viscosity ratio $N$ is large. These influence the onset of the FW by introducing a frequency-dependence of the instability threshold and critical wavenumber, as shown by Talib et al. (2007) in a combined experimental and theoretical study. Moreover, they found that the onset values are non-monotonic functions of $N$, so that increasing the viscosity ratio may promote instability.

Although the onset of the FW is well understood, the growth of the wave beyond onset has not been characterized. We are only aware of one study by Ivanova et al. (2001), which reports a series of wavelength measurements. Related wave formation at the interface between immiscible liquids of large viscosity contrast in a cylindrical geometry was investigated by Yoshikawa (2006) and Shyh \& 
Munson (1986), who performed wave height measurements suggesting onset through a supercritical pitchfork bifurcation. In this paper, we present the results of a detailed experimental investigation into the growth of the FW beyond onset as a function of the vibrational Froude number. As $h / l_{c}=21.35$ is large and our experimental evidence suggests that the layer height has a negligible effect on the wave growth, we choose $l_{c}$ as the characteristic length scale of the instability and define a modified Froude number based on $l_{c}, W=\widehat{W} \sqrt{h / l_{c}}$. Unlike most large-amplitude progressive waves, the wave in our experiments is symmetric about the vertical line passing through its crest at the mean position of the vessel (see figure 2). Thus, the wave shape at this location can be decomposed into a small number of Fourier components. Significant viscous dissipation in the upper layer liquid prevents the interfacial wave from developing instabilities through RIM, as suggested by McGoldrick (1970b). Hence, the growth of the FW enables the study of steep progressive capillary-gravity waves driven by shear. Note that sloshing is another mechanism of interfacial wave formation in horizontally oscillating fluid-filled tanks that is distinct from the FW. Strongly nonlinear waves may form when resonant conditions between the forcing and natural sloshing frequencies are approached (La Rocca, Sciortino \& Boniforti 2002), but in our geometry, these correspond to frequencies much smaller than those investigated in this paper.

The experimental apparatus, flow visualization and curvature measurement techniques are described in $\S 2$. In $\S 3.1$, we present bifurcation diagrams for the onset of the FW and show that the instability occurs through a supercritical pitchfork bifurcation. The qualitative change in the wave growth that occurs beyond a critical forcing $\left(W_{c}\right)$ is discussed in $\S 3.2$. The associated wave shape evolution is described and quantified in $\S 3.3$, and a transition from a weakly to a strongly nonlinear regime is identified to occur at $W_{c}$. In $\S 3.4$, the wavelengths in the strongly nonlinear regime are shown to depend on the forcing velocity $(a \omega)$ alone, whereas in the weakly nonlinear regime, an explicit dependence on the forcing frequency highlights the influence of viscosity. The effect of the contact line at the lateral walls of the vessel on the growth of the interfacial wave is addressed in $\S 3.5$. In $\S 4$, the transition between the two regimes is interpreted in terms of localized surface tension effects, and the effect of oscillation on wave growth and breaking is discussed. Finally, a summary of the results in given in $\S 5$.

\section{Experimental set-up}

The apparatus used to drive the flow is similar to that described in Talib et al. (2007). However, a new visualization set-up has been developed to enable the accurate observation of the shape of the interfacial wave by illuminating a thin slice of the interface using a sheet of laser light. Hence, we only highlight the salient features of the vibration rig, but discuss the visualization system and the measurement techniques in detail in $\S 2.1$ and $\S 2.2$, respectively.

A schematic diagram of the experimental apparatus is shown in figure 1(a). A rectangular Perspex box of inner dimensions $170 \mathrm{~mm} \times 75 \mathrm{~mm} \times 40 \mathrm{~mm}$ and $4 \mathrm{~mm}$ thick walls was filled with equal amounts of two immiscible liquids. A Perspex lid was fitted on top and care was taken to expel any air bubbles left inside the vessel. The box was mounted rigidly on a linear, horizontal air-bearing slide (Nelson Air), which was driven by a permanent-magnet shaker (LDS, V450). The harmonic content of the motion of the slide was less than $0.1 \%$ over the range of frequencies used in our investigation. The signal provided by the waveform generator (Agilent, 33440A) had a maximum error of $\pm 0.05 \mathrm{~Hz}$. Using feedback from a linearly variable differential 


$\begin{array}{ccc}\text { Lower layer } & v_{1}\left(\mathrm{~m}^{2} \mathrm{~s}^{-1}\right) & \rho_{1}\left(\mathrm{~kg} \mathrm{~m}^{-3}\right) \\ \text { Galden HT135 } & 1.12 \times 10^{-6} & 1752 \\ \text { Upper layer } & v_{2}\left(\mathrm{~m}^{2} \mathrm{~s}^{-1}\right) & \rho_{2}\left(\mathrm{~kg} \mathrm{~m}^{-3}\right) \\ \text { Silicone oil }(100 \mathrm{cS}) & 1.14 \times 10^{-4} & 961 \\ \text { Silicone oil }(200 \mathrm{cS}) & 2.10 \times 10^{-4} & 962\end{array}$

TABLE 1. Physical properties of the liquids used in the experiments. The surface tension coefficients between HT135 and the silicone oils were measured using a Du Nouy ring, and found to be equal for both silicone oils with a value of $\gamma=6.8 \times 10^{-3} \mathrm{~N} \mathrm{~m}^{-1}$.
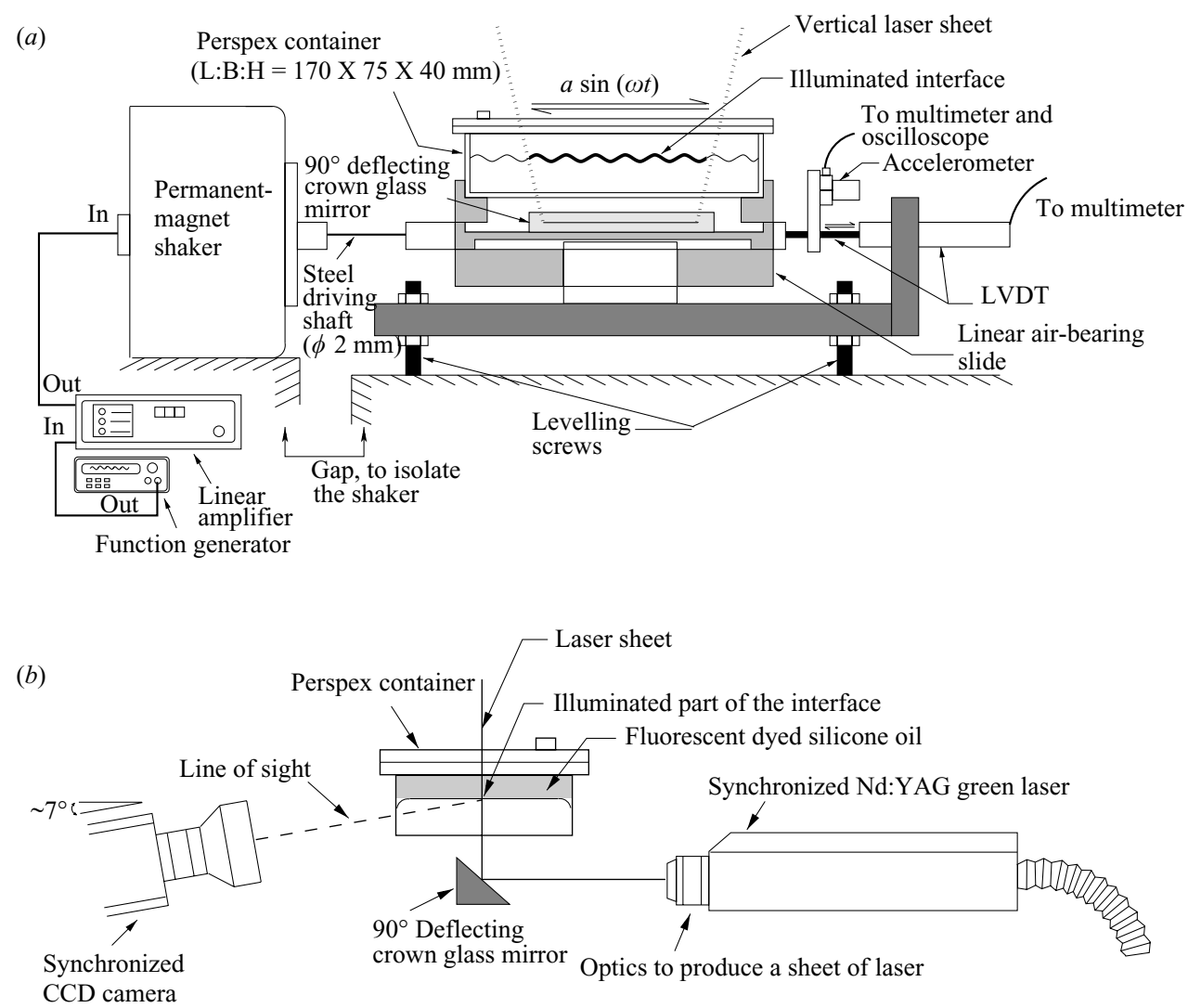

FIgURE 1. (a) Schematic diagram of the front view of the experimental set-up. (b) End view of the laser visualization set-up.

transducer (LVDT, Solatron, Mach 1), we could maintain the forcing amplitudes to within $0.1 \%$ of the set value. The external forcing was varied via two control parameters: the frequency and the amplitude of oscillation. The experiments were performed by fixing one of these quantities and varying the other. It was found experimentally that a settling time between parameter increments of approximately $30 \mathrm{~s}$ (i.e. between 600 and 900 oscillations) was sufficient to ensure the decay of transients.

The two immiscible liquids that we used in our experiments were Galden HT135 (a perfluoropolyether from Solvay Solexis) and silicone oil (polydimethylsiloxanes from Basildon Chemicals Ltd.). Two different silicone oils of kinematic viscosities of approximately $100 \mathrm{cS}$ and $200 \mathrm{cS}$ were used. The physical properties of the liquids 
are listed in table 1. They were measured in the laboratory at a temperature of $21^{\circ} \mathrm{C} \pm 1^{\circ} \mathrm{C}$, at which all the experiments were conducted. The fluids were chosen for their large density difference and low interfacial tension, in order to enable us to reach large-amplitude interfacial waves within the available power of the shaker. The maximum frequency for which waves of sufficient amplitude could be reached was $f=30 \mathrm{~Hz}$. The waves we observed in the experiments did not depend measurably on the transverse direction, and thus they were two-dimensional modes.

\subsection{Interface illumination}

The visualization of the interface was performed in the central part of the vessel in order to eliminate aberrations caused by the meniscus at the lateral walls. The line of contact between the fluid interface and the walls of the vessel was found to have a negligible effect on the wave dynamics as discussed in detail in §3.5. A laser sheet was shone in the vertical centre plane parallel to the direction of oscillations in order to illuminate a narrow slice of the interface. The contrast between the two fluid layers was maximized by dyeing the silicone oil with a fluorescent dye that is commercially available under the trade name Fluoro-Chek (Corrosion Consultants Div., USA). It fluoresces in the green and is commonly used for leak detection in automobile engines. This method resulted in a clean sharp interface. Moreover, the critical forcing parameters at the onset of the FW instability were found to be similar to within experimental resolution with and without the fluorescent dye present in the silicone oil, thus suggesting that the effect of the dye on the physical properties of the liquids was negligible.

A schematic diagram of the visualization set-up is shown in figure $1(b)$. The vertical laser sheet was produced by deflecting the horizontal light sheet from a pulsed Nd:YAG laser by $90^{\circ}$ using a slab of crown glass mirror positioned beneath the Perspex vessel. The camera was a Pulnix TM-6740, which has a resolution of $640 \times 480$ pixels. It was interfaced to a personal computer through a National Instruments (NI) PCI-1428 image capture card, and the NI Vision software was used to capture images. Both the camera and the laser were synchronized to the oscillations using a synchronizing digital pulse Transistor-Transistor Logic (TTL) from the waveform generator, in order to enable the stroboscopic capture of images. The TTL signal was modulated in phase before it reached the camera and the laser, so that the wave shape at different phases of the oscillations could be studied (see figure 2). The camera was positioned at a distance of approximately $300 \mathrm{~mm}$ from the vertical laser sheet with a positive inclination of approximately $7^{\circ}$ to the horizontal such that the line of sight ran through the transparent bottom liquid (figure $1 b$ ). In this way, the optical effects associated with the contact line were avoided entirely. This very small angle of inclination was chosen in order to minimize the magnification of the image caused by light refraction. The small residual effect of refraction was compensated for by calibrating the images in the plane of focus, which was displaced slightly from the illumination plane.

\subsection{Measurement techniques}

The growth of the FWs is accompanied by oscillations of the crests and troughs in the reference frame of the vessel. This means that in general, the waves are asymmetric about a vertical axis passing through their crests or troughs, except when the vessel is in its mean position (see figure 2). All of the measurements of the wave properties reported in this paper were taken at this mean position, where the waves are symmetric. For each set of forcing parameters, the wavelength and wave height were obtained by averaging the values of these quantities measured over three to 


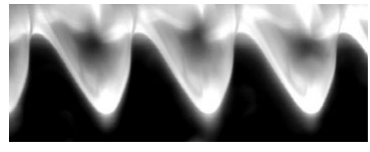

(a)

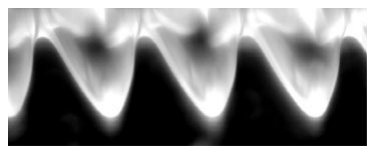

(b)

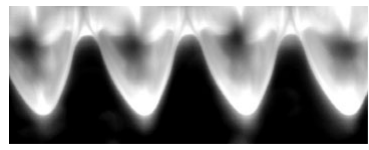

(c)

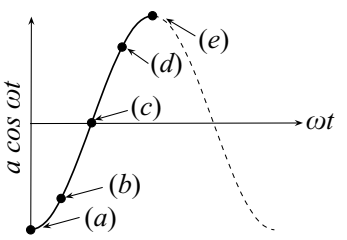

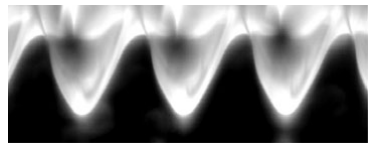

(d)

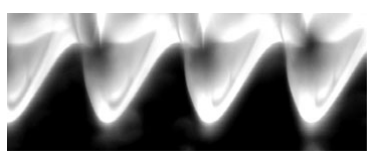

(e)

FIGURE 2. An illustration of the interface shape at the different phases of the oscillation cycle labelled on the schematic diagram of the vessel position. These pictures were captured with $200 \mathrm{cS}$ silicone oil in the upper layer. The waves in picture $(e)$ can be approximately recovered by reflecting the waves in picture $(a)$ about vertical lines passing through their crests or troughs, and similarly for the waves in pictures $(b)$ and $(d)$. The discussion of the interfacial wave in this paper is based on images of the vessel in its mean position, but the height and wavelength of the primary wave component remain similar to within experimental accuracy throughout the oscillation cycle.

five waves in ten successive images. The interface shape was very steady at a given phase of the oscillation cycle, so that the errors in wavelength measurements were within $\pm 0.5 \%$. The accuracy of the wave height measurements was limited by the resolution of the images, of approximately 30 pixels per millimetre. Thus, increasing uncertainties were unavoidable as the wave height decreased to infinitesimal values near onset, and the error on the smallest wave heights reported is up to $\pm 12 \%$.

The curvatures of the wave crests and troughs were measured by fitting secondorder polynomials to the outline of the wave crests and troughs using a least squares method, and calculating the maximum curvature of the fitted curves. Part of the wave near a crest or a trough was chosen for curvature measurements such that it lied within a square box of side equal to the capillary length (see $\S 4.1$ ), which corresponded to approximately 30 pixels in the digital images. The errors in these measurements were less than $4 \%$ if the width of the box was between 20 and 40 pixels. This method was validated by comparing the curvature values obtained at $f=30 \mathrm{~Hz}$ to the corresponding values obtained by fitting a truncated Fourier series (defined in (3.1)) to the wave shape, and the values were found to be similar.

The included angles of the wave crests $(\theta)$ were measured by drawing tangents on either sides of the crests such that the lines passed through their respective troughs, and measuring the angle between the two lines. The lines were drawn by hand, and the process was repeated three times for each image. The included angle measurement was taken to be the average of these three data points.

\section{Interfacial wave growth}

\subsection{Bifurcation diagram}

Bifurcation diagrams of the onset of the two-dimensional FWs are shown in figure $3(a)$, where the trough-to-crest height of the waves $\xi^{*}$ is plotted as a function of the amplitude of forcing, $a$. The measurements were made for forcing frequencies of $f=20 \mathrm{~Hz}, 25 \mathrm{~Hz}$ and $30 \mathrm{~Hz}$ (see $\S 2$ for the frequency range available), with 200 cS silicone oil in the upper layer. Similar bifurcation diagrams were obtained by 
(a)

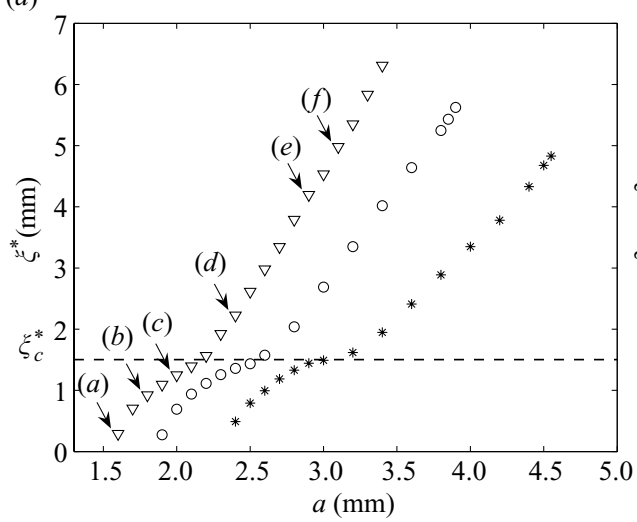

(b)

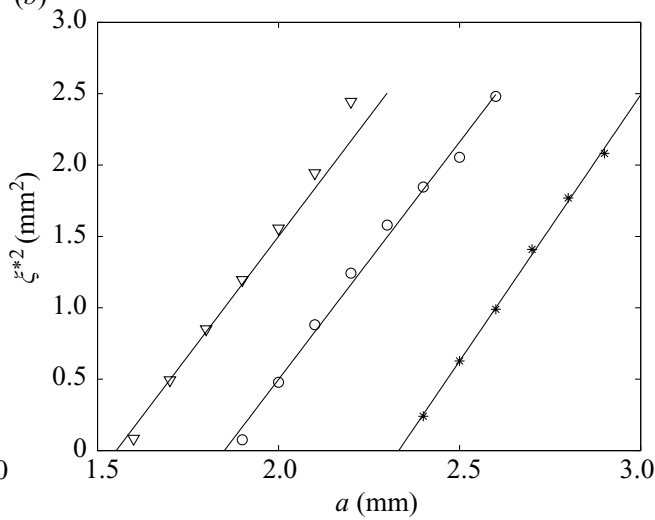

FIgURE 3. Bifurcation diagrams for different forcing frequencies of $f=20 \mathrm{~Hz}(*), f=25 \mathrm{~Hz}$ $(\bigcirc)$ and $f=30 \mathrm{~Hz}(\nabla)$, with $200 \mathrm{cS}$ silicone oil in the upper layer. The wave height, $\xi^{*}$, is plotted against the forcing amplitude $a$ in $(a)$. The labels on the bifurcation diagram refer to the pictures of the interface in figure 6. Plots of $\xi^{* 2}$ versus $a$ for data points below the dashed line in $(a)$ are plotted in $(b)$. Straight line fits are shown as solid lines.

varying $f$ as the bifurcation parameter while $a$ remained fixed. In figure $3(a)$, the dependence of $\xi^{*}$ on $a$ undergoes a qualitative change beyond a threshold height of the wave $\left(\xi_{c}^{*}\right)$, which is indicated by the horizontal dashed line in figure 3(a). For each value of the forcing frequency, the square of the heights $\left(\xi^{* 2}\right)$ below the horizontal dashed line depends linearly on $a$, as indicated by the linear least square fits plotted in solid lines in figure $3(b)$. Thus close to the point of onset of the FW, $a_{0}$, its height grows as $\sqrt{a-a_{0}}$. Furthermore, the same critical point $\left(a_{0}\right)$ is reached either by increasing or decreasing the forcing amplitude, indicating that there is no hysteresis at onset. These observations are consistent with a supercritical pitchfork bifurcation. The translational symmetry along the horizontal direction is broken for a critical value of the forcing by the deformation of the interface into waves (figure $6 a$ ). Note that we do not distinguish between different asymmetric states, and thus the symmetry-breaking bifurcation diagram includes only one branch.

The growth of the interfacial wave beyond $\xi_{c}^{*}$ is characterized by a linear variation of $\xi^{*}$ on $a$, and its slope increases with the forcing frequency (see figure $3 a$ ). The sudden qualitative change in the dependence of the wave height on the forcing parameters, when the wave height reaches $\xi_{c}^{*}$, suggests the existence of a transition from a weakly nonlinear to a strongly nonlinear state. We investigate the physical origin of this transition in $\S 4$.

\subsection{Dependence of the wave height on $W$}

The wave height, non-dimensionalized by the capillary length $\left(\xi=\xi^{*} / l_{c}\right)$, is plotted against the vibrational Froude number $\left(W=(a \omega) / \sqrt{g l_{c}}\right)$ in figure $4(a)$. The bifurcation diagram is divided into two regions (I and II), to highlight the square root and the linear variations of $\xi$ with $W$. In region $\mathrm{I}, \xi^{2}$ varies linearly with $W$ as shown in figure $4(b)$. However, the slopes of the linear fits to the data differ considerably, implying that the wave growth in region I is dependent on $f$ or $a$ individually, rather than on the product (af). This is an effect of viscosity that is discussed in further detail in §3.4. By contrast in region II, the four curves collapse onto a straight line to within experimental uncertainty suggesting a dependence on $(a f)$ only. 


$\begin{array}{ccccc}f(\mathrm{~Hz}) & 18.9 & 20 & 25 & 30 \\ a(\mathrm{~mm}) & 3.500 & 3.216 & 2.625 & 2.160 \\ W_{c} & 4.359 & 4.215 & 4.301 & 4.247\end{array}$

TABLE 2. Values of $W_{c}$ obtained for different experimental parameters from data of figure 4. In the first column, $a$ was fixed and the forcing frequency $(f)$ was varied, so the intersection point of the square root and linear fits gives a critical value of $f$. The experiments reported in the following columns were for fixed frequencies, so that $a$ was varied.

(a)

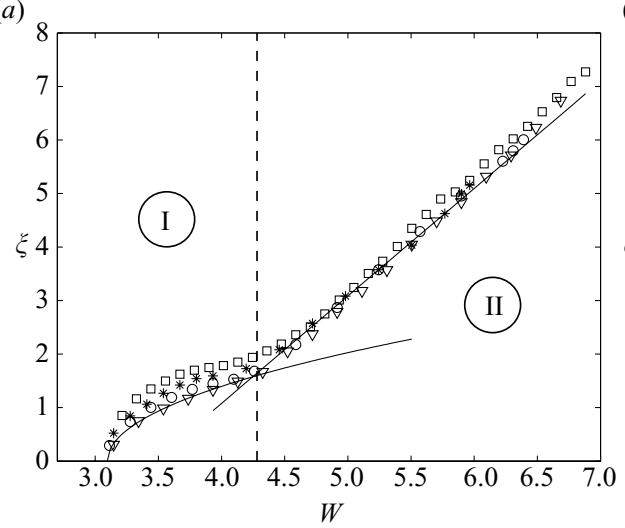

(b)

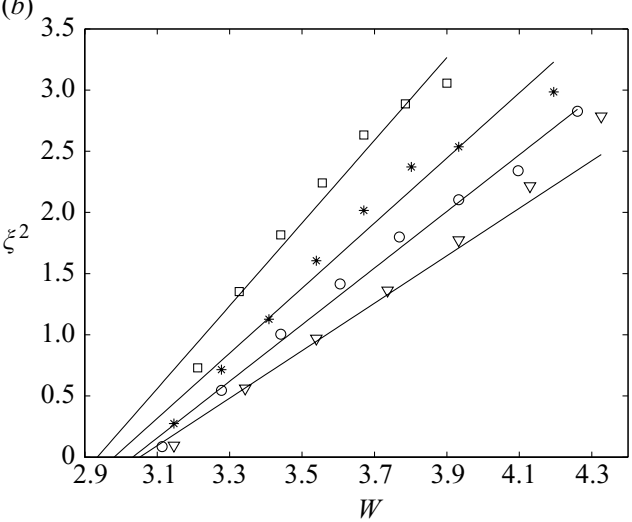

Figure 4. ( $a$ ) Wave height $(\xi)$ versus $W$ for different fixed parameters; $f=20 \mathrm{~Hz}(*), f=25 \mathrm{~Hz}$ $(\bigcirc), f=30 \mathrm{~Hz}(\nabla)$ and $a=3.5 \mathrm{~mm}(\square)$, with $200 \mathrm{cS}$ silicone oil in the upper layer. Regions I and II are demarcated by the vertical dashed line at $W_{c}=4.2807$. In region II, all the data points collapse onto a straight line with an average slope of $1.938 \pm 0.068$. The average linear fit and the square root fit for $f=30 \mathrm{~Hz}$ are shown with solid lines. (b) Square of the wave height $\left(\xi^{2}\right)$ versus $W$ in region I for different fixed parameters. The linear fits to each data set are shown with solid lines.

For each set of data, a critical value of the Froude number $W_{c}$ was determined to be the intersection point of the square root and linear fits in regions I and II, respectively. Close to $W_{c}$, the weighted norm of the residues for the square root fit increased sharply, so that points included in the square root fit were those up to the value of $W$ beyond which an order of magnitude jump in this quantity was observed. The rest of the points were included in the straight line fit in region II. The average slope of the straight line fits in region II is $1.938 \pm 0.068$, which is constant to within $\pm 3.5 \%$. Despite the different square root fits in region I (figure $4 b$ ), the values of $W_{c}$ tabulated in table 2 differ by only $1.4 \%$ with an average of 4.281 , which can be considered constant to within experimental accuracy. Hence, the data collapse for $W \geqslant W_{c}$.

A comparison between the bifurcation diagrams measured with $100 \mathrm{cS}$ and $200 \mathrm{cS}$ silicone oils is shown in figure 5 . With $100 \mathrm{cS}$ silicone oil, $W_{c}=4.379 \pm 0.006$. This value is only $2.28 \%$ larger than the result obtained with $200 \mathrm{cS}$ sillicone oil, compared with the $14 \%$ difference between the forcing values at the onset of the FWs, $W_{\mathrm{o}}$. Moreover, the critical heights of the waves $\left(\xi_{c}=\xi_{c}^{*} / l_{c}\right)$ are approximately equal in both cases. This is not an effect of the finite thickness of the fluid layers, as even the tallest wave heights are less than $15 \%$ of the layer thickness. The fact that the 


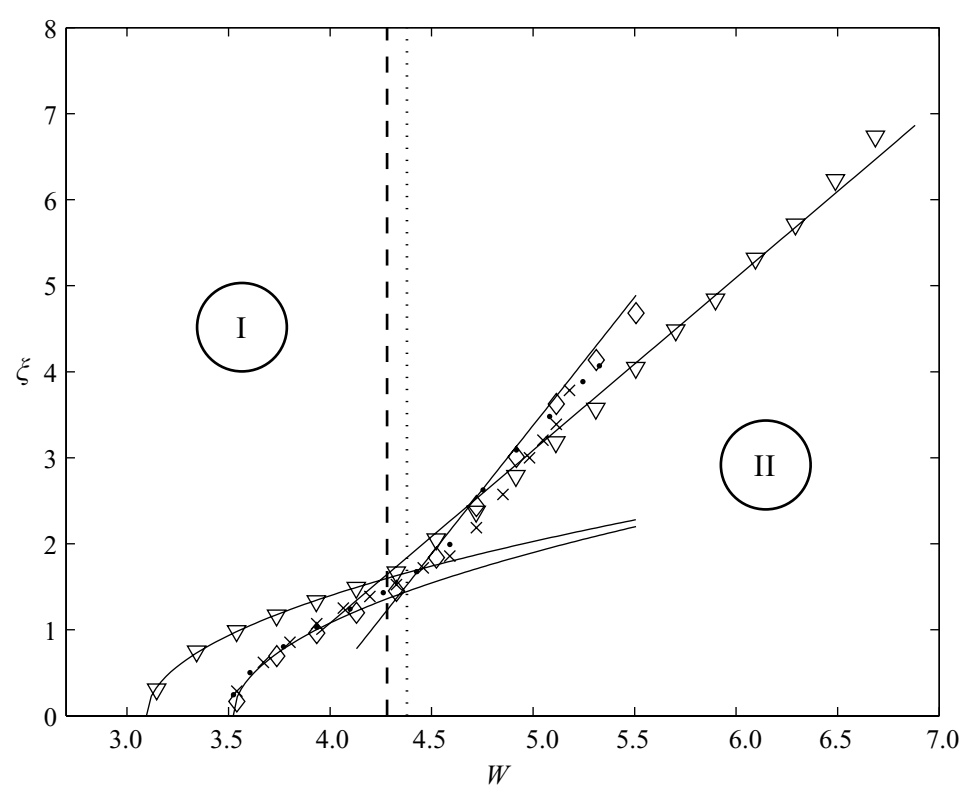

FIGURE 5. Comparison between wave heights when the upper layer liquids are $200 \mathrm{cS}$ and $100 \mathrm{cS}$ silicone oils. The values of $W_{c}$, highlighted by dashed and dotted vertical lines, respectively, are very close. The data taken with $100 \mathrm{cS}$ silicone oil was for forcing frequencies of $f=20 \mathrm{~Hz}(\cdot), f=25 \mathrm{~Hz}(\times)$ and $f=30 \mathrm{~Hz}(\diamond)$. The square root fits for $f=30 \mathrm{~Hz}$ and average linear fits for different fixed amplitudes and frequencies of forcing for each liquid are shown with solid lines.

wave heights in region II vary linearly with different slopes, however, suggests that the dependence on the viscosity ratio $(N)$ is sustained beyond $W_{c}$.

\subsection{Description of wave shape evolution}

In figure 6, we present a set of six pictures of the interface that illustrate the evolution of the interfacial wave shape with forcing amplitude for $f=30 \mathrm{~Hz}$ and $200 \mathrm{cS}$ silicone oil. These pictures correspond to the points marked with letters on the bifurcation diagram in figure $3(a)$. The pictures in the left hand-side column of figure 6 are for $W_{\mathrm{o}}<W<W_{c}$, while the right hand-side column is for $W>W_{c}$. The interface undergoes successive qualitative changes as $W$ is increased. The sinusoidal shape of the interface in figure 6(a) evolves to resemble an inverted trochoid in figure 6(c). As $W$ is increased beyond $W_{c}$, it deforms further to assume a 'finger'-like crest as shown in figures $6(e)$ and $6(f)$.

The excellent agreement between linear theory and the experiments of Talib et al. (2007) suggests that the interfacial wave is sinusoidal at the onset of the FW instability, although very close to onset, the waves have vanishingly small heights, so that their shape cannot be resolved experimentally. The curvatures of the crests and troughs of the wave are equal at onset, but as the wave grows, the trough curvature increases more steeply than the crest in the region $W_{\mathrm{o}}<W \leqslant W_{c}$. Hence we see sharper troughs than crests in figure $6(a-c)$. In these three pictures, the curvatures of the crests (troughs), non-dimensionalized by the capillary length, are $(a) 0.2(0.23)$, (b) 0.62 (1.07), (c) 0.95 (1.91), respectively. The differences in curvature of the crests and troughs are accentuated as the forcing frequency is decreased, and we refer to $\S 4.1$ for more detailed curvature measurements. 
(a)
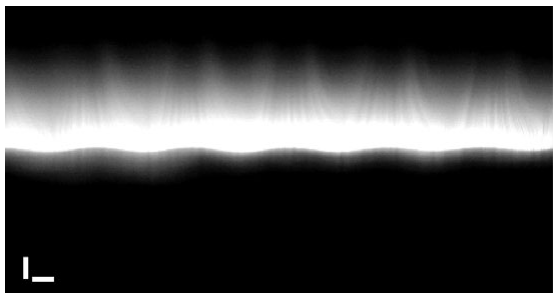

(b)

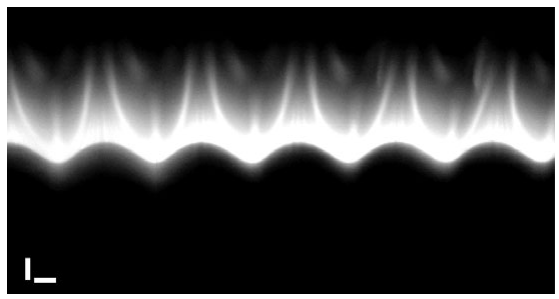

(c)

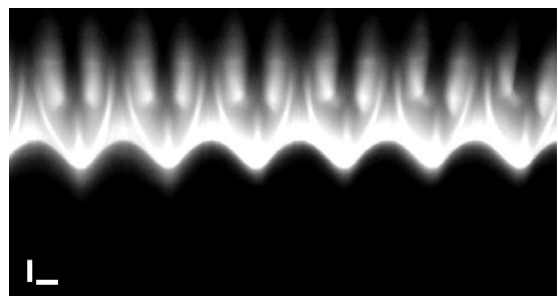

(d)

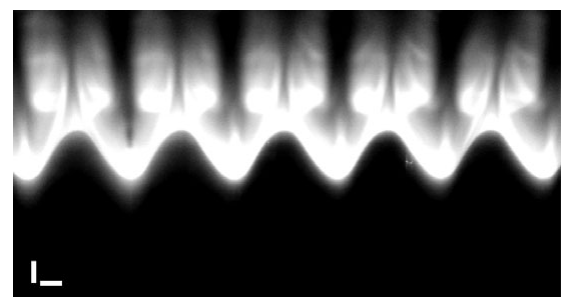

(e)

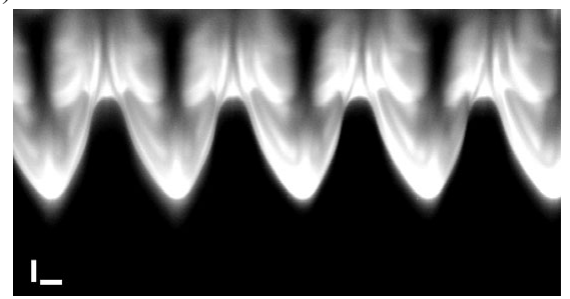

(f)

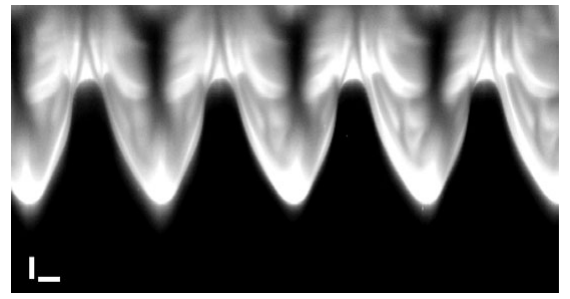

FIGURE 6. Evolution of the interfacial wave shape at $f=30 \mathrm{~Hz}$ and with $200 \mathrm{cS}$ silicone oil for the increasing amplitudes of forcing highlighted in figure 3. (a) The interface shape at $W=3.14$ is close to sinusoidal. In $(b) W=3.51$ and $(c) W=3.93$, the interface adopts an inverted-trochoid shape. In $(d)(W=4.71)$, the trough starts to broaden, while in $(e)(W=5.68)$ and $(f)(W=6.10)$, the crest has developed a 'finger'-like shape. The white bars in the lower left corner of each image correspond to the capillary length scale $\left(l_{c}\right)$.

When the height of the wave reaches $\xi_{c}$ (at $W_{c}$ ), the shape of the wave changes qualitatively. The curvature of the trough starts to decrease, while the crest curvature continues to increase (see figure $12 a$ ) but at a lower rate. Hence for $W>W_{c}$, the trough broadens but its depth continues to increase analogously to the wave height. This results in the deformation of the crest to a 'finger'-like shape that accommodates the broadening as well as the deepening of the trough (figure $6 f$ ). The physical origin of these changes will be discussed in $\S 4$.

The nonlinear evolution of the interfacial wave is quantified by analysing the harmonic content of the wave shape. Using a least squares method, we fit the truncated Fourier series,

$$
y=A_{0} \cos (k x)+A_{1} \cos (2 k x)+A_{2} \cos (3 k x),
$$

to the interfacial wave shape extracted from the experimental images, and determine the magnitudes of the fundamental $\left(A_{0}\right)$, first $\left(A_{1}\right)$ and second $\left(A_{2}\right)$ harmonic terms. $x$ and $y$ are the horizontal and vertical coordinates, respectively, $k$ is the wavenumber measured in the experiments, and these quantities are non-dimensionalized with the capillary length. The first three terms of the Fourier expansion are sufficient to 
(a)

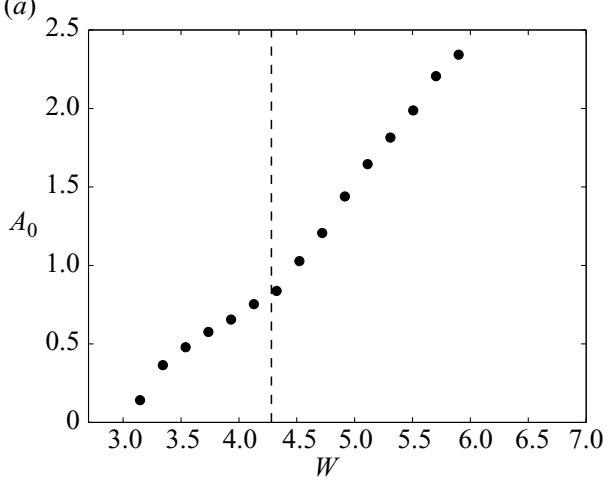

(b)

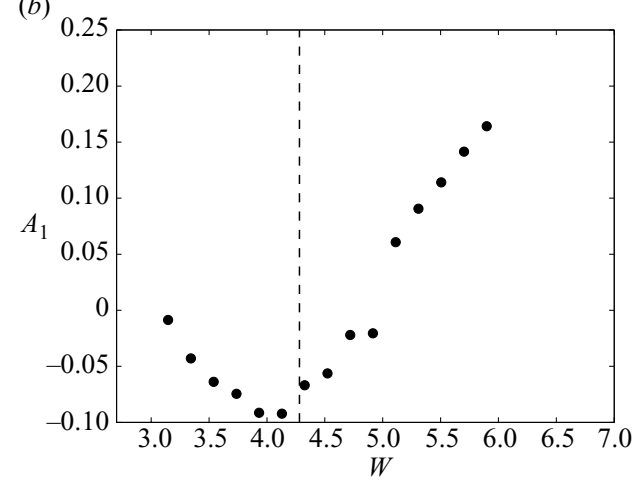

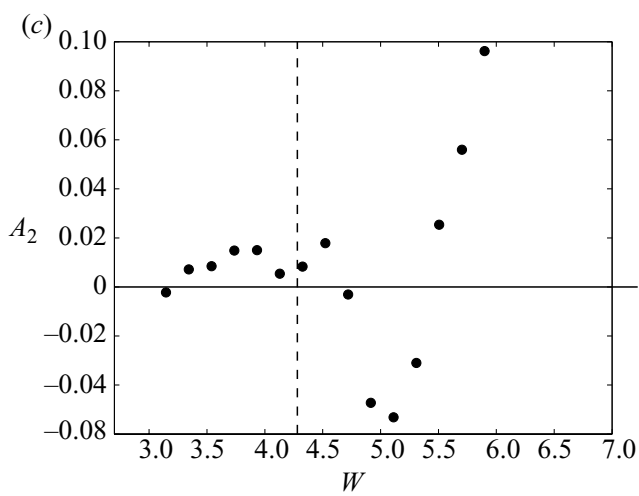

FIGURE 7. Variation of the amplitudes $A_{0}, A_{1}$ and $A_{2}$ from (3.1) with $W$. The experiments were performed by varying $a$ for $f=30 \mathrm{~Hz}$, with $200 \mathrm{cS}$ silicone oil in the upper layer. The dashed vertical lines highlight the value of $W_{c}$.

describe the trochoid shape and the 'fingering' of the crest, and thus, we have not included higher order terms in (3.1). Moreover, the magnitude of the fourth term in the series is less than 0.01, which is approximately an order less than those of the second and third terms.

In figure 7, we plot the variation of $A_{0}, A_{1}$ and $A_{2}$ for $f=30 \mathrm{~Hz}$ with $200 \mathrm{cS}$ silicone oil in the upper layer. The fundamental mode is approximately an order of magnitude larger than the first harmonic, and hence it exhibits a similar variation with $a$ as the trough-to-crest height $(\xi)$ plotted in figure $4(a)$. The first harmonic affects the curvatures of crests and troughs in opposite ways. The negative values taken by $A_{1}$ for $W \leqslant W_{c}$ imply that the first harmonic acts to increase the curvature of the troughs and decrease the curvature of the crests. The decrease in $A_{1}$ towards its minimum value at approximately $W_{c}$ results in the inverted trochoid profile of the interface seen in figure $6(c)$. The second harmonic, however, affects both curvatures in a similar way. $A_{2}$ is approximately equal to zero for $W \leqslant W_{c}$ and drops to negative values immediately beyond $W_{c}$, so that it acts to moderate the curvatures of both the crests and troughs. The cumulative contributions of both the first and second harmonics lead to the narrowing of the crest into a 'finger'. Examples of the inverted trochoid shape and the 'finger'-shaped crest are shown in figure 8, where the outlines of these two interfacial waves, plotted with dashed lines, are superposed onto sine waves of the corresponding wavenumber $k$ and wave height $2\left(A_{0}+A_{1}+A_{2}\right)$. The qualitative changes in the harmonic content of the wave at $W_{c}$ 
(a)

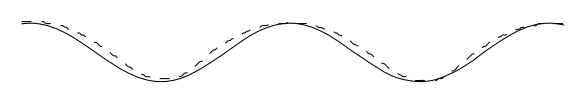

(b)

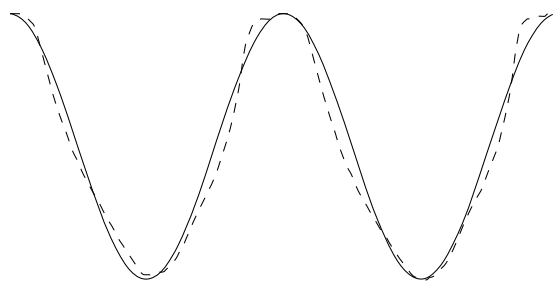

FIGURE 8. Experimental wave shapes, with $200 \mathrm{cS}$ silicone oil in the upper layer, that deviate from the sinusoidal form are plotted with a dashed line. The solid lines give the sinusoidal wave form with a wavelength and wave amplitude taken from the experiments. ( $a$ ) A typical wave from region I, with broader crests and narrower troughs (inverted trochoid). (b) The 'finger' like shape of the wave crest seen in region II.

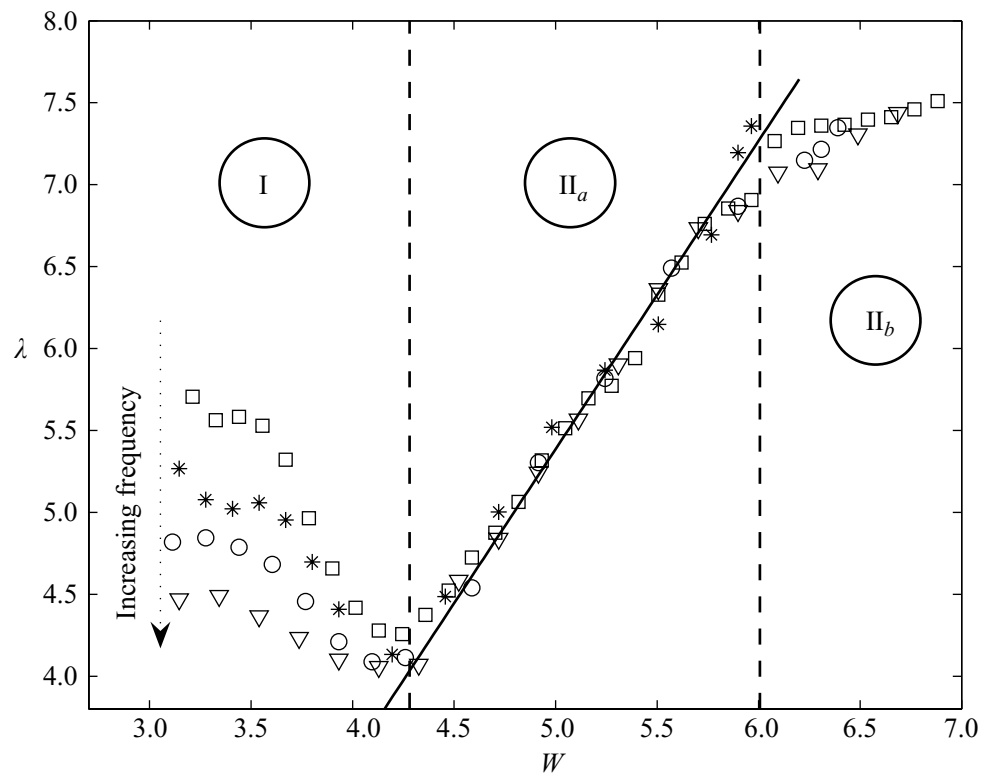

FIGURE 9. Plot of the non-dimensional wavelength $(\lambda)$ versus $W$ for the same parameters as in figure 4. The dotted arrow indicates that the onset wavelengths decrease with increase in forcing frequency $(f)$. In region II, the wavelengths collapse and increase steeply with $W$. The wavelengths in regions $\mathrm{II}_{a}$ and $\mathrm{II}_{b}$ increase at different rates. The solid line is the linear fit for the wavelength data corresponding to $f=30 \mathrm{~Hz}$ in region $\mathrm{II}_{a}$.

further support the existence of a transition from a weakly nonlinear state to a strongly nonlinear state.

\subsection{Dependence of the wavelength on $W$}

The variation of the non-dimensional wavelength $\left(\lambda=\lambda^{*} / l_{c}\right)$ with $W$ for $200 \mathrm{cS}$ silicone oil is shown in figure 9. The wavelength successively decreases and increases for $W<W_{c}$ and $W>W_{c}$, respectively, thus reaching a minimum at $W=W_{c}$. Similarly to the wave height variation, the wavelengths collapse onto a master curve for $W \geqslant W_{c}$. For $W<W_{c}$, however, the wavelength decreases with increasing forcing frequency, as highlighted by the dotted arrow. 


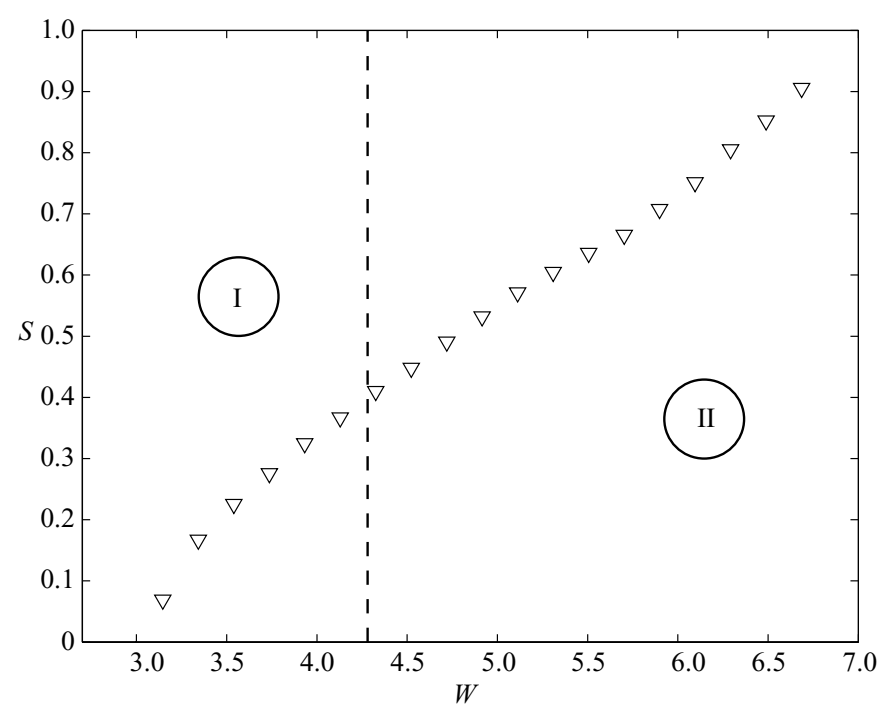

Figure 10. The steepness $\left(S=\xi^{*} / \lambda^{*}\right)$ of the two-dimensional waves versus the forcing $W$ as the wave approaches the onset of three-dimensional waves for $f=30 \mathrm{~Hz}$ and $200 \mathrm{cS}$ silicone oil. For larger forcing, $S \sim O(1)$, which implies that $\xi^{*} \sim \lambda^{*}$.

By fitting a power law to the experimental data of Talib et al. (2007), we obtain a functional dependence of the critical wavelength of primary instability on $f$ for $N=187$, which shows that at onset $\lambda^{*} \propto f^{-0.31}$. For $f=30 \mathrm{~Hz}$, the thickness of the Stokes layers are $\delta_{2}=0.4 \lambda^{*}$, and $\delta_{1}=0.03 \lambda^{*}$ at the onset of the FWs. The fact that these length scales, and particularly $\delta_{2}$, are close to the value of $\lambda^{*}$ suggests that viscous effects have a strong influence on the wavelength selection. If $\delta_{2}$ is used to non-dimensionalize the wavelength instead of $l_{c}$, then $\lambda=\lambda^{*} / \delta_{2}=\lambda^{*} \sqrt{\pi / \nu_{2}} f^{1 / 2}$. This indicates $\lambda^{*} \propto f^{-0.5}$, and hence suggests a frequency dependence of wavelength due to viscous effects in the upper layer. However, since this power of $f$ is not close to the experimentally observed power of -0.31 at onset, other factors such as the viscous effects in the lower layer are likely to influence the wavelength dependence on $f$.

In region II, we find that the wavelength is a function of the product of amplitude and frequency of forcing, but looses its explicit dependence on $f$. This suggests that viscous stresses in the Stokes layers do not influence the shape of the interfacial wave in this region. We expect the surface tension forces to have a more significant effect on the wavelength in this region since the radius of curvature associated with local features of the shape of the nonlinear interfacial waves become comparable to $l_{c}$, as discussed in $\S 4$.

The variation of the wavelength and wave height with the magnitude of forcing in this region may be inferred by balancing inertial and gravitational forces, as inertial forces induce suction at the crests and troughs that tends to deform the interface, while gravitational forces act to restore a flat interface. For larger magnitudes of forcing, e.g. in region II, the wave steepness, defined as the ratio of the wave height to wavelength $\left(S=\xi / \lambda=\xi^{*} / \lambda^{*}\right)$, tends towards one (figure 10). Hence, the wave height and wavelength are of the same order $\left(\xi^{*} \sim \lambda^{*}\right)$. The balance of forces for a wave of dimensional wavelength $\lambda^{*}$ can be expressed as $\frac{\rho_{1}+\rho_{2}}{2}(a \omega)^{2}\left(\xi^{*} / \lambda^{*}\right) \lambda^{*} \sim\left(\rho_{1}-\rho_{2}\right) g \xi^{*} \lambda^{*}$, 


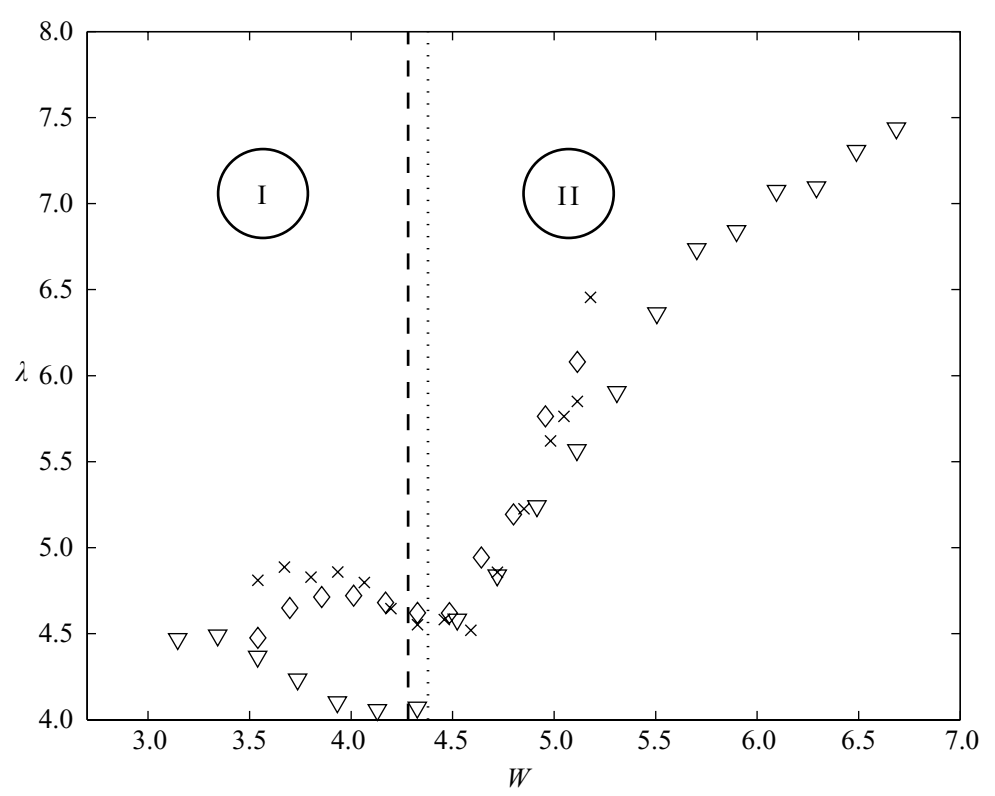

FIGURE 11. Wavelength versus $W$ for experiments performed with $200 \mathrm{cS}$ and $100 \mathrm{cS}$ silicone oil in the upper layer. The data points from the $100 \mathrm{cS}$ silicone oil experiments are for forcing frequencies of $f=20 \mathrm{~Hz}(\times)$, and $f=30 \mathrm{~Hz}(\diamond)$. The data points from the $200 \mathrm{cS}$ silicone oil experiments are for $f=30 \mathrm{~Hz}(\nabla)$. The values of $W_{c}$ for $100 \mathrm{cS}$ and $200 \mathrm{cS}$ silicone oil experiments are indicated by dotted and dashed vertical lines, respectively.

which yields

$$
\frac{\lambda^{*}}{l_{c}} \sim \frac{\xi^{*}}{l_{c}} \sim \frac{W^{2}}{\Delta \rho}
$$

where $\Delta \rho=2\left(\rho_{1}-\rho_{2}\right) /\left(\rho_{1}+\rho_{2}\right)$. This relation suggests that the wavelength and wave height vary as the square of the vibrational Froude number in region II. However, we find that the linear fits in $W$ and $W^{2}$ are of similar precision because of the limited range of $W$ where steep waves are observed. The maximum value of $W$ in this regime is set by the onset of three-dimensional instability (see $\S 4.2$ ). Hence, we cannot conclusively establish the functional dependence of wavelength on the forcing parameter, and have chosen to show the lowest order fit for the wave height and wavelength plots in figures 4 and 9 , respectively. Note that the equally good fits for $\lambda$ versus $W$ and $W^{2}$ data sets is not a result of simply considering a higher order polynomial, because we do not include the $W$ term while fitting the data for $W^{2}$. However, surface tension forces are of the same order as gravitational forces in this regime, so that they contribute to define the wave shape locally and may alter the relationship between wave properties and vibrational Froude number.

The influence of the other viscosity parameter $N$ is more involved, as indicated by the non-monotonic variation of the onset wavelengths with $N$ (Talib et al. 2007). A comparison between wavelengths measured in experiments with $100 \mathrm{cS}$ and $200 \mathrm{cS}$ silicone oil are shown in figure 11. Similarly to the wave height comparison shown in figure 5, the wavelengths for these two different viscosity ratios differ considerably in region I. In region II, the influence of $N$ is still noticeable, although the wavelengths for both values of the viscosity ratio only exhibit small differences, similarly to the wave heights. Hence, in region II, $N$ may act on the wave by influencing the average 
velocity difference between the layers, but does not impact the wave shape, which is primarily determined by surface tension effects as discussed in $\S 4$.

Finally, a more detailed examination of figure 9 reveals that region II can itself be divided into two regions, $\mathrm{II}_{a}$ and $\mathrm{II}_{b}$. The steep growth of the wavelength in region $\mathrm{II}_{a}$ is followed by saturation in region $\mathrm{II}_{b}$, which is linked to the deformed contact lines observed for large $W$ (see $\S 4.2$ ). This behaviour is consistent with the results of Ivanova et al. (2001), who report a 'jump' in the wavelength variation with forcing frequency when the constant forcing amplitude is relatively small.

\subsection{Effect of the contact line on the wave growth}

Talib et al. (2007) have shown that the line of contact between the liquid interface and the lateral walls of the container has a negligible effect on the onset of the instability in the experiments. The contact line exhibits slip-stick motion, such that it remains at rest for small deformations of the interface, and starts to move above a critical deformation (Dussan 1979). As the interfacial wave grows, the deformation of the interface caused by the oscillating crests within a cycle of external forcing always remains below the critical value required to make the contact line slip. Hence, for any fixed set of parameter values, the contact line does not deform within the oscillation cycle. Slip, however, may occur during the transient evolution associated with changes of parameter values, resulting in a wavy contact line. A deformed contact line is not observed until values of $W$ in region II, and thus it does not affect the value of $W_{c}$. Furthermore, when the forcing was increased from the onset of the primary instability to the maximum value shown in figure 9, and then reduced back to a value for which the interface is flat, we found similar variations of $\lambda$ with $W$, with only slight differences in wavelengths in the region of steepest variation beyond $W_{c}$, for all the fixed values of amplitude or frequency investigated. As advancing contact angles (when increasing the forcing) are generally different from receding contact angles (when decreasing the forcing) (Dussan 1979), any effect of the contact line is expected to contribute to hysteresis in the wavelength diagram. The small changes observed in the wavelength plot suggest that the effect of the contact line on the dynamics of the interface is minimal.

\section{Surface tension effects}

\subsection{Curvature measurements}

In figure $12(a)$, the dimensionless radius of curvature of the crest $\left(r_{\kappa}=r_{\kappa}^{*} / l_{c}\right)$ is plotted as a function of $W$ for different forcing frequencies. The horizontal solid line highlights the value of the capillary length. $r_{\kappa}$ decreases rapidly in region I and reaches the capillary length at approximately $W_{c}$. On this length scale, both gravity and surface tension restoring forces are of similar magnitude and thus contribute equally to shape the interface. In region I where $r_{\kappa} \gg 1$, the wave is gravity-dominated, whereas in region $\mathrm{II}_{a}$ surface tension has a stronger effect on the wave shape relative to region I since $r_{\kappa} \sim 1$, and even slightly larger than the gravitational force as $r_{\kappa} \lesssim 1$. In broad terms, the gravitational force acts to reduce the volume of fluid displaced by the formation of the interfacial wave, which is proportional to the wavelength, resulting in the decrease of wavelengths in region I. The surface tension force acts to reduce curvature, and thus promotes an increase in the wavelength in region $\mathrm{II}_{a}$.

The increased influence of surface tension forces in region $\mathrm{II}_{a}$ is also apparent in the dimensionless curvature plots shown in figure $13(a)$ for $f=30 \mathrm{~Hz}$ and $200 \mathrm{cS}$ silicone oil, where the curvatures of the troughs decrease for $W>W_{c}$. However, both crest 
(a)

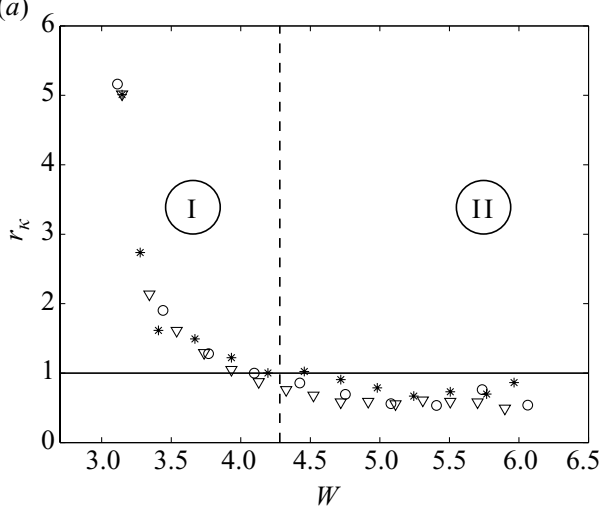

(b)

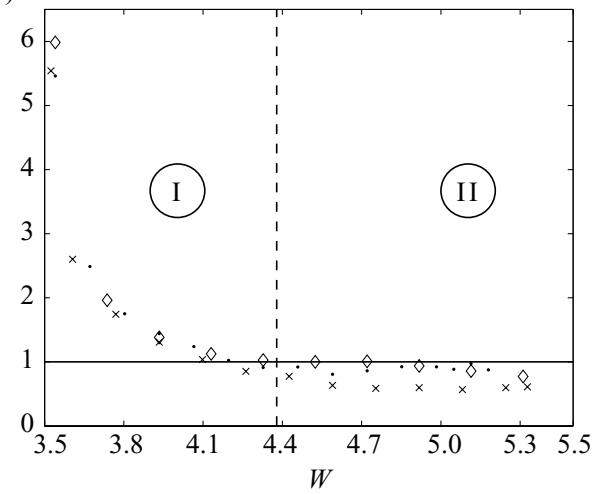

Figure 12. (a) Radius of curvature values of the crest $\left(r_{\kappa}\right)$ for $f=20 \mathrm{~Hz}(*), f=25 \mathrm{~Hz}(\bigcirc)$ and $f=30 \mathrm{~Hz}(\nabla)$ versus $W$ for the $200 \mathrm{cS}$ silicone oil experiments. $r_{\kappa} \sim 1$ very close to $W_{c}$ for the different curves. (b) Similarly, for $100 \mathrm{cS}$ silicone oil experiments, the radius of curvature values for the trough $\left(r_{\kappa}\right)$ intersects the value of the capillary length of 1 close to $W_{c}$ for $f=20 \mathrm{~Hz}(\times), f=25 \mathrm{~Hz}(\cdot)$ and $f=30 \mathrm{~Hz}(\diamond)$.

(a)

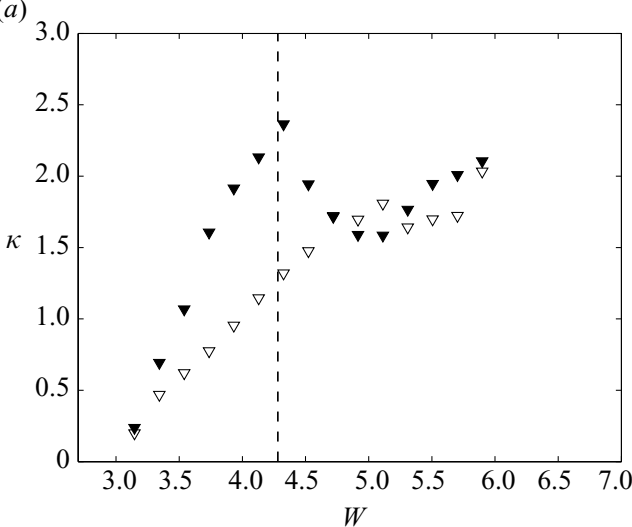

(b)

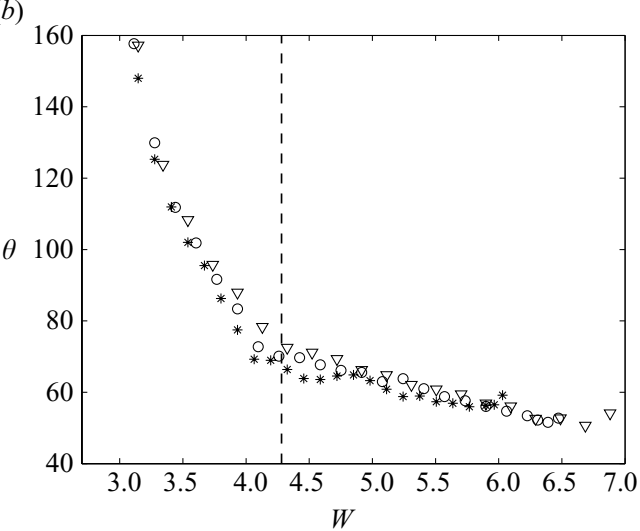

FiguRE 13. (a) The curvatures of the crests and troughs, $\left(\kappa=\kappa^{*} l_{c}\right)$, increase at different rates. For $W \geqslant W_{c}$, the curvature of the trough $(\boldsymbol{\nabla})$ decreases while that of the crest $(\nabla)$ continues to increase but at a slower rate. $(b)$ Included angle of the interfacial wave $(\theta)$ in degrees, versus $W$ for $f=20 \mathrm{~Hz}(*), f=25 \mathrm{~Hz}(O)$ and $f=30 \mathrm{~Hz}(\nabla)$. The curvature and included angle data are for $200 \mathrm{cS}$ silicone oil in the upper layer.

and trough curvatures retain values above 1 as $W$ increases. These variations lead to the formation of 'finger'-like crests, which have smaller curvatures than the sinusoidal crests, but are also narrower so that they allow the curvatures of the troughs to decrease. Note in figure $13(a)$ that the transition to strongly nonlinear wave does not occur until the smaller of the two curvatures, i.e. that of the crest for experiments in $200 \mathrm{cS}$ silicone oil, has reached the capillary length. In the experiments with $100 \mathrm{cS}$ silicone oil, it is the curvature of the trough that is smaller than that of the crest, and hence reaches the capillary length at $W=W_{c}$. In this case, the shape of the wave in region $\mathrm{I}$ is a trochoid by contrast with the inverted trochoid observed in the $200 \mathrm{cS}$ oil experiments (see figure 8). These findings suggest that the value of the capillary length plays a crucial role in determining $W_{c}$, and that the transition at $W_{c}$ is only weakly dependent on the viscosity of the upper layer liquid. 


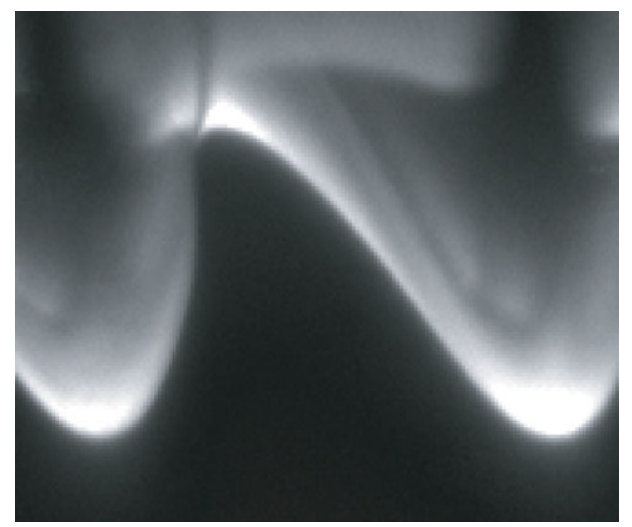

FIGURE 14. Image of a wave close to an extreme position of the oscillation cycle whose crest is overhanging. $f=20 \mathrm{~Hz}$ and with $200 \mathrm{cS}$ silicone oil in the upper layer.

Hence, the transition from weak to strong surface tension effect appears to be determined by the local features of the wave shape. Similar effects have been observed in steep standing water waves by Schultz et al. (1998). Furthermore, the shape of our interfacial waves in region I resemble the weakly nonlinear gravity waves described by McGoldrick (1970a). Note, however, that the gravity waves of McGoldrick have a trochoid shape, whereas in our experiments, they exhibit either trochoid or invertedtrochoid shapes, depending on the viscosity of the upper layer. The variation of the qualitative features of the wave with viscosity ratio indicates that interfacial shear influences the relative sharpness of the crests and troughs, as suggested by Thorpe (1978). Reduced interfacial shear in the case of $N=100$ means a smaller influence on the wave-shape, so that the trochoid nature of the gravity-dominated waves is preserved. The difference in curvature between the crests and troughs decreases with the increase of the forcing frequency as discussed in $\S 3.4$. Increased shear due to an increase in $f$, on the contrary, makes the wave shape more trochoidal, in contrast with the results of Thorpe (1978). Hence, further investigation is required to understand the observed change in the wave shape with variation in $N$.

\subsection{Maximum wave heights}

The variation of the included angle $(\theta)$ with $W$ is plotted in figure $13(b)$ for $200 \mathrm{cS}$ silicone oil. We see that the values of $\theta$ for all forcing frequencies beyond $W_{c}$, are approximately equal to $\theta=60 \pm 10^{\circ}$. When the included angle saturates for $W>W_{c}$, we do not observe wave breaking as in the weakly nonlinear Stokes wave. Instead, the wave shape evolves from an inverted-trochoid (weakly nonlinear state) to a more complicated shape with its characteristic 'finger'-like crest (strongly nonlinear state), and this enables the wave height to continue to increase, as previously discussed in $\S 3.2$ and $\S 3.3$.

When the vessel is displaced from its mean position, the interface shape tends towards an 'S' configuration (see figure 14) that would be unstable to Rayleigh-Taylor instability under static conditions. However, the wave does not break for the range of forcing frequencies and amplitudes explored in our study. The largest horizontal velocity associated on the wavy interface has to exceed the phase speed for that region to overhang (Holyer 1979), and our observations suggest that this condition is satisfied before the vessel reaches its maximum displacement. As the vessel decelerates as it approaches the maximum displacement position, the wave is not subject to unstable 
conditions for sufficient time for the wave to overturn irreversibly. This shows that the prediction of Meiron \& Saffman (1983) of the existence of overhanging waves is experimentally realizable by employing sinusoidal forcing.

Finally, we find that the wave height continues to increase with $W$ in region II until the wave becomes unstable to three-dimensional travelling waves, which emanate from the side walls and propagate towards the centre of the vessel.

\section{Conclusion}

We have presented an experimental study of the nonlinear growth of capillarygravity waves at the interface between two immiscible liquids subject to horizontal oscillations. They are driven by a K-H instability and arise through a supercritical pitchfork bifurcation. The sinusoidal forcing prevents the waves from overturning, thus enabling the study of steep waves. From the Fourier decomposition of our measured interfaces, we have identified a critical value of the vibrational Froude number $W_{c}$ at which the wave undergoes a transformation from a weakly nonlinear state $\left(\left|A_{2}\right| \sim 0\right)$ to a strongly nonlinear state $\left(\left|A_{2}\right| \neq 0\right)$, where $A_{2}$ is the amplitude of the second harmonic component.

This transformation is accompanied by qualitative changes in the variation of the wave height and wavelength with $W$. The bifurcation curves undergo a qualitative change from square root to linear variations with $W$ at $W_{c}$. In the weakly nonlinear regime (region $\mathrm{I}$ ), the wavelengths decrease at different rates depending on the value of the fixed experimental parameter, $a$ or $f$, and reach a minimum at $W=W_{c}$. The wavelength looses its explicit dependence on $f$ for $W \geqslant W_{c}$, by collapsing onto a single straight line with a positive slope. This indicates that the waves are influenced by viscous length scales for $W<W_{c}$.

The physical explanation for the qualitative changes that occur at $W_{c}$ stems from the observation that the radius of curvature of the crest (or trough in the case of $100 \mathrm{cS}$ silicone oil) $r_{\kappa}^{*}$ becomes equal to the capillary length $\left(l_{c}\right)$ at approximately $W_{c}$. This implies that at $W_{c}$, surface tension and gravitational forces acting on the wave crests are of the same magnitude.

As even large-amplitude wave shapes at the mean position of the vessel are accurately described by a truncated Fourier series of only three significant terms, a time-averaged analytical description could be envisaged. Numerical calculations, however, would probably be required to establish the nature of the transition from weakly to strongly nonlinear waves.

We wish to thank L. Limat, R. E. Hewitt, A. L. Hazel and T. Mullin for fruitful discussions. This work was funded by an Overseas Research Scholarship (SVJ) and an EPSRC 'Advanced Research Fellowship' (AJ).

\section{REFERENCES}

Chandrasekhar, S. 1981 Hydrodynamic and hydromagnetic stability. Dover.

Cox, C. S. 1958 Measurements of slopes of high-frequency wind waves. J. Mar. Res. 16, 199-225.

Craik, A. D. D. 1985 Wave interactions and fluid flows. Cambridge University Press.

Crapper, G. D. 1957 An exact solution for progressive capillary waves of arbitrary amplitude. J. Fluid Mech. 2, 532-540.

Drazin, P. G. 1970 Kelvin-Helmholtz instability of finite amplitude. J. Fluid Mech. 42, 321-335.

Dussan, E. B. 1979 On the spreading of liquids on solid surfaces: static and dynamic contact lines. Annu. Rev. Fluid Mech. 11, 371-400. 
GonZÁLEZ-Viñas, W. \& SALÁn, G. 1994 Surface waves periodically excited in a $\mathrm{CO}_{2}$ tube. Europhys. Lett. 26, 665-670.

Helfrich, K. R. \& Melville, W. K. 2006 Long nonlinear internal waves. Annu. Rev. Fluid Mech. 38, 395-425.

HolYer, J. Y. 1979 Large amplitude progressive interfacial waves. J. Fluid Mech. 93, 433-448.

Hou, T. Y., Lowengrub, J. S. \& Shelley, M. J. 1997 The long-time motion of vortex sheets with surface tension. Phys. Fluids 9, 1933-1954.

Ivanova, A. A., Kozlov, V. G. \& EvesQue, P. 2001 Interface dynamics of immiscible fluids under horizontal vibration. Fluid Dyn. 36, 362-368.

Jiang, L., Perlin, M. \& Schultz, W. W. 1998 Period tripling and energy dissipation of breaking standing waves. J. Fluid Mech. 369, 273-299.

Khenner, M. V., Lyubimov, D. V., Belozerova, T. S. \& Roux, B. 1999 Stability of plane-parallel vibrational flow in a two-layer system. Eur. J. Mech. - B/Fluids 18, 1085-1101.

KraskovskiI, Y. P. 1960 On the theory of steady waves of not small amplitude (in Russian). Dokl. Akad. Nauk SSSR 130, 1237.

La Rocca, M., Sciortino, G. \& Boniforti, M. A. 2002 Interfacial gravity waves in a two-fluid system. Fluid Dyn. Res. 30, 31-66.

Longuet-Higgins, M. S. 1963 The generation of capillary waves by steep gravity waves. J. Fluid Mech. 16, 138-159.

Lyubimov, D. V. \& Cherepanov, A. A. 1987 Development of a steady relief at the interface of fluids in a vibrational field. Fluid Dyn. 86, 849-854.

MCGoldRICK, L. F. 1970a An experiment on second-order capillary gravity resonant wave interactions. J. Fluid Mech. 40, 251-271.

McGoldrick, L. F. $1970 b$ On Wilton's ripples: a special case of resonant interactions. J. Fluid Mech. 42, 193-200.

Meiron, D. I. \& Saffman, P. G. 1983 Overhanging interfacial gravity waves of large amplitude. J. Fluid Mech. 129, 213-218.

Phillips, O. M. 1988 Remote sensing of the sea surface. Annu. Rev. Fluid Mech. 20, 89-109.

Schultz, W. W., Vanden-Broeck, J. M., Jiang, L. \& Perlin, M. 1998 Highly nonlinear standing waves with small capillary effect. J. Fluid Mech. 369, 273-299.

Shyн, C. K. \& Munson, B. R. 1986 Interfacial instability of an oscillating shear layer. J. Fluid Eng. 108, 89-92.

Stokes, G. G. 1847 On the theory of oscillatory waves. Camb. Phil. Soc. Trans. 8, 441-455.

TALib, E., JALIKoP, S. V. \& Juel, A. 2007 The influence of viscosity on the frozen wave instability: theory and experiment. J. Fluid Mech. 584, 45-68.

Talib, E. \& Juel, A. 2007 Instability of a viscous interface under horizontal oscillation. Phys. Fluids 19, 092102.

TAYLOR, G. I. 1953 An experimental study of standing waves. Proc. R. Soc. Lond. A 218, 44-59.

THORPE, S. A. 1978 On the shape and breaking of finite amplitude internal gravity waves in a shear flow. J. Fluid Mech. 85, 7-31.

Wolf, G. H. 1969 The dynamic stabilization of the Rayleigh-Taylor instability and the corresponding dynamic equilibrium. Z. Physik 227, 291-300.

Wunenburger, R., Evesque, P., Сhabot, C., Garrabos, Y., Fauve, S. \& Beysens, D. 1999 Frozen wave instability by high frequency horizontal vibrations on a $\mathrm{CO}_{2}$ liquid-gas interface near the critical point. Phys. Rev. E 59, 5440-5445.

Yoshikawa, H. N. 2006 Instabilitiés des interfaces, sous oscillations. PhD Thesis, Université Paris 6. 